\title{
COMPARATIVE EVALUATION OF BACTEC 460TB SYSTEM AND LOWENSTEIN-JENSEN MEDIUM FOR THE ISOLATION OF M. TUBERCULOSIS FROM CEREBROSPINAL FLUID SAMPLES OF TUBERCULOUS MENINGITIS PATIENTS
}

\author{
MM Venkataswamy, W Rafi, S Nagarathna, V Ravi, *A Chandramuki
}

\begin{abstract}
Purpose: To evaluate the role of the radiometric BACTEC 460TB system and the conventional Lowenstein-Jensen (LJ) medium for isolation of M. tuberculosis from cerebrospinal fluid (CSF) samples of tuberculous meningitis (TBM) patients. Methods: CSF specimens $(\mathrm{n}=2325)$ from suspected TBM patients were processed for isolation of mycobacteria by inoculating BACTEC 12B medium and the LJ medium. The isolation of mycobacteria in both media was confirmed by microscopy and biochemical identification. Drug sensitivity testing for the anti-TB drugs was carried out by BACTEC radiometric method. Results: Among the total 2325 CSF specimens processed by both methods, M. tuberculosis was isolated from 256 specimens. The isolation rates were $93 \%$ and $39 \%$ for the BACTEC system and LJ medium respectively. Both the media supported growth in $32 \%$ of the culture-positive specimens. BACTEC system alone yielded growth in $61 \%$ and $\mathrm{LJ}$ alone in 7\%, of the culture-positive specimens. Among 205 isolates tested for drug susceptibility $81 \%$ were sensitive to all the drugs tested and 19\% were resistant. Conclusions: The BACTEC 460TB system provides a highly sensitive and rapid tool for the isolation and drug susceptibility testing of $M$. tuberculosis, from CSF of TBM patients. Use of a solid medium in conjunction with the BACTEC 12B medium is essential for optimal recovery for M. tuberculosis from CSF specimens.
\end{abstract}

Key words: BACTEC radiometric method, cerebrospinal fluid, Lowenstein-Jensen medium, sensitivity, tuberculous memingitis

Tuberculous meningitis (TBM) is the most malignant form of neurotuberculosis. ${ }^{1}$ Case fatality rate of untreated TBM is almost $100 \%$ and delay in treatment may lead to permanent neurological damage. ${ }^{2}$ Microscopy by Ziehl-Neelsen (ZN) staining of cerebrospinal fluid (CSF) is positive in less than $10 \%$ of cases and isolation by using conventional solid media are associated with low sensitivity and is time consuming. The nucleic acid assays, owing to low sensitivity and high costs, are only an adjunct to and not the substitute for the conventional procedures. ${ }^{3}$ The Centre for Disease Control and Prevention (CDC) Atlanta, Georgia, USA guidelines recommend that the laboratory identification of the $M$. tuberculosis complex be accomplished within 14-21 days after specimen collection. ${ }^{4}$ Liquid media, as demonstrated by the radiometric BACTEC 460 TB system (BectonDickinson, Towson, MD) appear to be the best alternative for this purpose. It has evolved as the "gold standard" by which all other culture and detection systems are evaluated. ${ }^{5}$ This

*Corresponding author (email: <acmuki@gmail.com>)

Departments of Neuromicrobiology (WR, SN, AC) and Neurovirology (VR), National Institute of Mental Health and Neurosciences, Bangalore - 560 029, India; and Department of Microbiology and Immunology (MMV), Albert Einstein College of Medicine, Bronx, NY-10461, USA

Received: 29-12-06

Accepted: 15-02-07 system uses a selective broth medium containing C14-labelled palmitic acid, which improves recovery and decreases the time required for detection of mycobacteria. ${ }^{6-10}$ The BACTEC mean detection time of $M$. tuberculosis in smear negative specimens has been reported to be better for tissues and fluids (14 days) than for respiratory specimens (24 days). ${ }^{11}$ Recent recommendations for optimal recovery of mycobacteria from clinical specimens are to use a liquid medium along with one or more solid media, with Lowenstein-Jensen (LJ) slants being recommended as the primary medium. ${ }^{12}$ Although the BACTEC 460TB system has been shown to be very useful in the diagnosis of pulmonary TB there are few published reports evaluating its role in the isolation of mycobacteria from extra pulmonary specimens, especially CSF specimens. We report the results of a study carried out to assess the utility of BACTEC radiometric method in the diagnosis of TBM in comparison with $\mathrm{LJ}$ medium and to determine the drug susceptibility testing of CSF isolates of $M$. tuberculosis.

\section{Materials and Methods}

\section{Clinical specimens}

During the period January 2000 to December 2003, 2325 CSF specimens from suspected TBM patients were received for mycobacterial culture. All specimens were inoculated simultaneously into the BACTEC 12B broth (BectonDickinson, USA) and the LJ medium (Hi-Media, India). 


\section{Culture procedures}

Sterile, uncentrifuged CSF samples were directly inoculated without decontamination. One each of LJ medium slope and BACTEC 12B vial was inoculated for each CSF specimen.

About $0.5-1 \mathrm{~mL}$ of CSF was added to the BACTEC 12B medium. As recommended, $0.1 \mathrm{~mL}$ of PANTA supplement (polymixin B $50 \mu \mathrm{g} / \mathrm{mL}$, amphotericin B $5 \mu \mathrm{g} / \mathrm{mL}$, nalidixic acid $20 \mu \mathrm{g} / \mathrm{mL}$, trimethoprim $5 \mu \mathrm{g} / \mathrm{mL}$ and azlocillin $10 \mu \mathrm{g} / \mathrm{mL}$. Becton Dickinson) was added to each BACTEC 12B vial prior to specimen inoculation. The culture media were incubated at $37^{\circ} \mathrm{C}$, for six weeks. The BACTEC vials were tested for growth every two days during the first two weeks and weekly thereafter. BACTEC cultures showing a growth index $(\mathrm{GI}) \geq 10$ were read daily until the achievement of GI 50-100 and were subjected to Ziehl-Neelsen (ZN) stain and Gram stain. The vials, which remained negative for six weeks, were discarded. ${ }^{13}$

A slope of LJ medium was inoculated with 0.25 to 0.5 $\mathrm{mL}$ of each specimen and incubated at $37^{\circ} \mathrm{C}$ for eight weeks. The LJ slants were inspected weekly. Growth on the LJ slants resembling mycobacterial colonies i.e., rough, tough and buff-coloured, was subjected to $\mathrm{ZN}$ staining and Gram staining to confirm the presence of acid-fast bacilli and rule out contamination respectively.

\section{Identification}

The pure BACTEC mycobacterial cultures were tested by the BACTEC NAP ( $p$-nitro- $\alpha$-acetylamino- $\beta$ hydroxypropiophenone) test to differentiate $M$. tuberculosis complex from mycobacteria other than tubercle (MOTT) bacilli. ${ }^{13,14}$ The NAP vial was inoculated with $1 \mathrm{~mL}$ of the culture from a positive 12B vial. Both the vials were incubated at $37^{\circ} \mathrm{C}$ and tested daily for four to six days on the BACTEC instrument and the GI of the NAP vial as well as the control vial were recorded. The isolates were identified as belonging to $M$. tuberculosis complex when the NAP vial showed two consecutive significant decreases in GI or slight increase in the first two days and then a decrease or no increase. The final identification of all the cultures from LJ medium was done at the National Tuberculosis Institute, Bangalore.

\section{Drug susceptibility testing}

Once identified as an isolate of M. tuberculosis complex by the NAP test, the original positive vial was incubated till it reached a GI of 500-800, when the culture was used for sensitivity testing. If the GI was greater than 800 it was diluted 1:2 in the BACTEC diluting fluid prior to sensitivity testing. From the stock solutions of the primary line drugs $0.1 \mathrm{~mL}$ was added to four different $12 \mathrm{~B}$ vials to achieve final concentrations of streptomycin $2 \mu \mathrm{g} / \mathrm{mL}$, isoniazid 0.1 $\mu \mathrm{g} / \mathrm{mL}$, rifampicin $2 \mu \mathrm{g} / \mathrm{mL}$ and ethambutol $2.5 \mu \mathrm{g} / \mathrm{mL}$. Into each of these drug vials, $0.1 \mathrm{~mL}$ of the well-mixed culture was inoculated. The control vial was inoculated with $0.1 \mathrm{~mL}$ of the culture, diluted 1:100, in BACTEC diluting fluid. This method is equivalent to the proportion method carried out using LJ medium, which detects $1 \%$ or more of the drugresistant population. The BACTEC 12B vials with the drugs and the control vial were tested in the BACTEC instrument to introduce $5 \% \mathrm{CO}_{2}$ and then incubated at $37^{\circ} \mathrm{C}$. The vials were read daily for a minimum of five days and a maximum of 12 days. On the day the control vial reached a GI of 30 or more, the difference in GI values from the previous day, designated $\Delta \mathrm{GI}$, were calculated for all the vials. If the $\Delta \mathrm{GI}$ of the drug vial was equal to or more than that of the control vial, the result was reported as resistant and if $\Delta$ GI was less than the control vial it was reported as sensitive. ${ }^{13}$

\section{Results}

\section{Mycobacterial isolation}

Of the total 2325 specimens inoculated into BACTEC and LJ media, 256 yielded growth of mycobacteria. Among these culture positive specimens, BACTEC 12B medium could successfully support growth in 237 (93\%) CSF specimens and LJ medium facilitated mycobacterial growth from 101(39\%) of the specimens (Fig. 1). All the 237 isolates from BACTEC 12B belonged to the $M$. tuberculosis complex based on the NAP differentiation test. All the isolates from LJ medium were identified to be $M$. tuberculosis by performing the niacin test, paranitro-benzoic acid test and the nitrate-reduction test. Eightytwo $(32 \%)$ of the 256 culture positive specimens yielded growth in both media, while $155(61 \%)$ specimens yielded growth only in the BACTEC 12B medium as against $19(7 \%)$ only on LJ medium (Fig. 1). The average number of days required to detect growth by the radiometric method was 15 days, while it was 35 days by the $\mathrm{LJ}$ medium culture. The contamination rate for BACTEC $460 \mathrm{~TB}$ was $4 \%$ and that for LJ medium was $10 \%$.

\section{Drug susceptibility}

Drug susceptibility testing was performed for 205 of 256

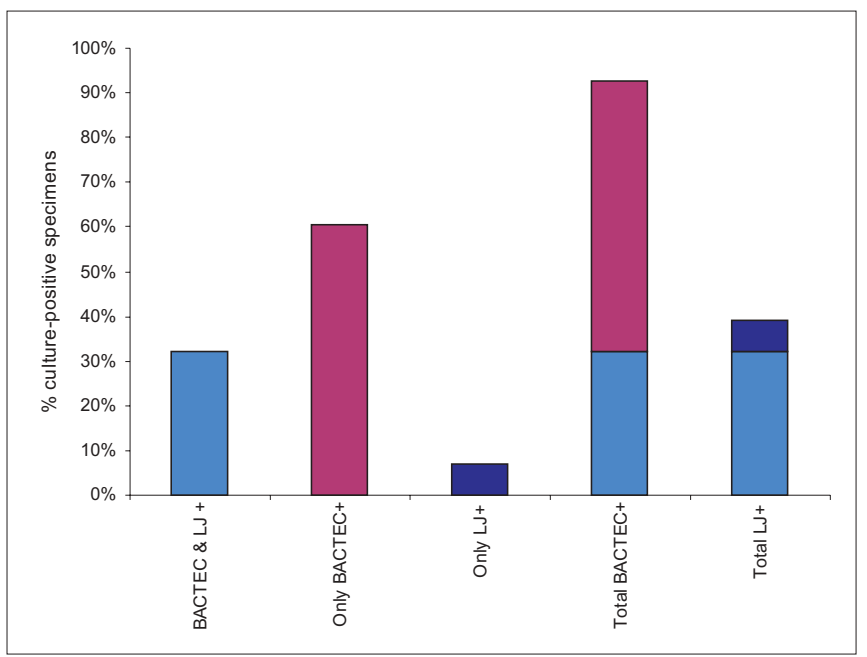

Figure 1: Comparison of isolation rates between BACTEC 12B and LJ medium culture among the culture positive CSF specimens $(n=256)$ 
isolates. Most of the isolates, $166(81 \%)$, were sensitive to all the four drugs tested i.e., streptomycin (STM), isoniazid (INH), rifampicin (RIF) and ethambutol (EMB). Drug resistance was recorded in 39(19\%) of the tested isolates (Fig. 2). Single drug resistance to INH was observed in $27(13 \%)$ isolates. Five isolates $(2 \%)$ were resistant to both INH and STM. There were other solitary isolates with varied resistance patterns as shown in the Table. Multidrug resistance, as defined by resistance to INH and RIF, was detected in three $(1.5 \%)$ isolates.

\section{Discussion}

This study was carried out to evaluate the utility of BACTEC $12 \mathrm{~B}$, in conjunction with the conventional LJ medium, in the early diagnosis of TBM. The sensitivity and rapidity of detection of mycobacterial growth in the CSF specimens from TBM patients was compared between the two different media and the role of BACTEC method in rapid drug susceptibility testing was also assessed. The data from this study shows that the rate of recovery of mycobacteria from

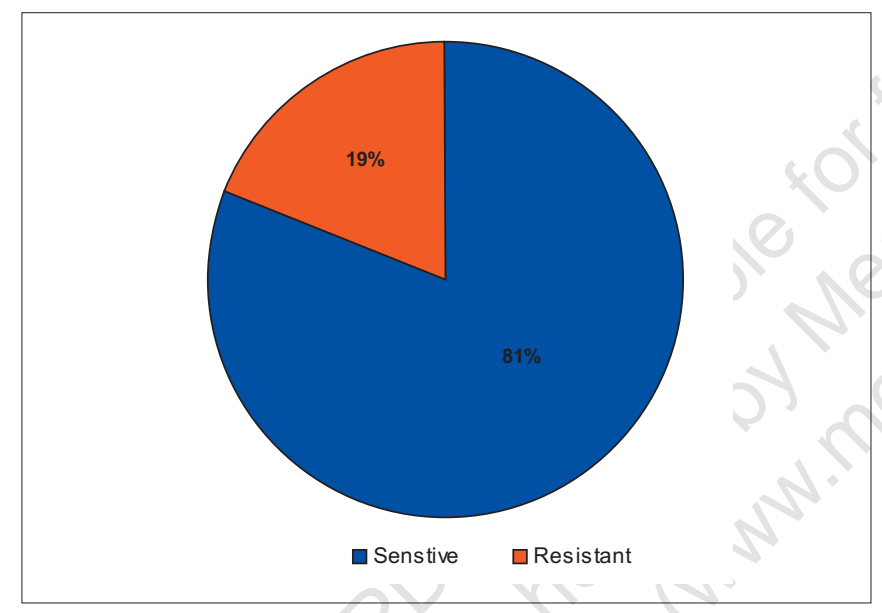

Figure 2: Rate of drug sensitivity and resistance in the CSF isolates $(\mathrm{n}=205)$ of $M$. tuberculosis

Table: Drug susceptibility patterns of $M$. tuberculosis isolates $(n=205)$ from cerebrospinal fluid of tuberculous meningitis patients

\begin{tabular}{lc}
\hline Drug susceptibility patterns & No. of isolates (\%) \\
\hline Susceptible to STM, INH, RIF, EMB & $166(81)$ \\
Resistant to INH & $27(13)$ \\
Resistant to STM & $1(0.5)$ \\
Resistant to EMB & $1(0.5)$ \\
Resistant to STM and INH & $5(2)$ \\
Resistant to INH and RIF* & $1(0.5)$ \\
Resistant to INH and EMB & $1(0.5)$ \\
Resistant to STM, INH and RIF* & $1(0.5)$ \\
Resistant to STM, INH and EMB & $1(0.5)$ \\
Resistant to INH, RIF and EMB* & $1(0.5)$ \\
Total & $\mathbf{2 0 5}$ \\
\hline
\end{tabular}

INH - Isoniazid, STM - Streptomycin, EMB - Ethambutol, RIF Rifampicin, *Multidrug resistant
CSF specimens by BACTEC radiometric method is far more superior as compared to the conventional culture on $\mathrm{LJ}$ medium. Moreover, the radiometric method is superior to the solid medium culture even in terms of rapidity in detection of growth. The recovery rates varying from 80 to $95 \%$ have been reported using BACTEC 12B whilst for LJ medium it varies from 42 to $77 \% .^{12}$ The recovery rate in our study using BACTEC $12 \mathrm{~B}$ was $93 \%$, as against $39 \%$ by $\mathrm{LJ}$ medium. Thus the recovery rates in our study are consistent with observations from previous reports. ${ }^{12}$ Among the total 2325 CSF specimens that were processed by both methods, 256 isolates grew in either media or both of them. Although $32 \%$ among these 256 culture positive specimens yielded growth in both the culture media, $61 \%$ were positive only in BACTEC 12B, which clearly demonstrates the enhanced sensitivity provided by this automated system in the laboratory diagnosis of TBM. There were $7 \%$ of the culture-positive specimens, which yielded growth only in the LJ medium. Therefore, there was no single medium, which could enable the detection of all the culture positive specimens. This suggests that some of the M. tuberculosis positive specimens may fail to yield growth if no additional solid medium is incorporated along with BACTEC 12B. Therefore the BACTEC system must be used concurrently with conventional culturing methods. ${ }^{15}$ The reasons for some of the isolates which grew only on LJ and not in BACTEC 12B could be that these isolates were unable to metabolize the palmitic acid in this medium ${ }^{16}$ or some of the growth promoting factors present in the egg-based medium may be absent in the BACTEC 12B medium. All the isolates belonged to the $M$. tuberculosis Complex as identified by the NAP differentiation test. In this study, only one additional solid medium was included with BACTEC 12B based on an earlier report, which compared the performance of BACTEC 12B, LJ medium and Middlebrookes 7H11 medium for isolation of mycobacteria from clinical specimens. The results of this earlier study suggested that there was no significant difference in the recovery of mycobacteria irrespective of whether an LJ slant or Middlebrooks 7H11 was used as an additional solid medium with BACTEC 12B medium. ${ }^{10}$

Drug susceptibility test was performed by the BACTEC radiometric method for $205 \mathrm{CSF}$ isolates of $M$. tuberculosis. The results were available within three weeks after identification of mycobacterial growth in the 12B medium by $\mathrm{ZN}$ staining. The drug susceptibility patterns suggest that most of the TBM cases are still caused by strains that are sensitive to anti tubercular drugs. Our data showed $80.98 \%$ of the clinical isolates to be sensitive to the first line drugs STM, INH, RIF and EMB. Therefore, by clinching an early diagnosis and with prompt treatment, it is possible to significantly bring down the high rates of mortality and morbidity associated with this dreaded form of extra-pulmonary TB. Resistance to INH was the most common (13.17\%) single-drug resistance recorded which is consistent with earlier reports. Multi-drug resistance, i.e., resistance to INH and RIF, was observed in only three $(1.46 \%)$ isolates, suggesting that MDR-TB is still not a confounding problem in the management of neurotuberculosis. 
However, with a tremendous escalation in the incidence of $\mathrm{HIV}$-associated TB and recently reported incidence of TBM due to MDR strains, ${ }^{17}$ it is essential to closely monitor the incidence of MDR-TB of CNS by regularly testing the drug susceptibility of the clinical isolates.

In addition to the higher rate of recovery another significant advantage in using the BACTEC 12B was in terms of the time required for the detection of mycobacterial growth. The time for detection of growth by BACTEC method was twice as quick as that taken by LJ medium, an observation that is consistent with earlier reports. ${ }^{11,18}$ The BACTEC mean detection time of $M$. tuberculosis in smear-negative specimens was better for tissues and fluids (14 days) than for respiratory specimens (24 days). ${ }^{11}$ The average time for recovery by BACTEC $12 \mathrm{~B}$ was two weeks as against four to five weeks by LJ medium. In fact, many a times the isolation, identification and drug sensitivity testing was completed by the BACTEC method by the time growth could be detected on LJ medium. Another merit of BACTEC 12B medium was the lower number of specimens lost due to contamination i.e. $4 \%$ against a contamination rate of $10 \%$ on $\mathrm{LJ}$ medium. Therefore, the BACTEC 12B medium provides a tremendous advantage over LJ medium in terms of its rapidity in growth detection, increased sensitivity and a lower rate of specimens lost due to contamination. ${ }^{19}$ Moreover, automated procedures carry the potential of introducing standardization in the mycobacteriology laboratory. ${ }^{18}$

The biggest hurdle associated with the use of this method in a developing country is the initial investment and the recurrent costs that are involved. By facilitating early diagnosis, the BACTEC method may prove to be cost effective in a population with a high prevalence of tuberculosis, particularly in the extrapulmonary and paucibacillary forms of the disease. ${ }^{6,19}$ The importance of rapid detection and identification of mycobacteria for the management of TBM, which is the most malignant form of extrapulmonary TB, MDR-TB and HIV-associated TB, far outweighs the problems encountered due to cost constraints. However, the application of non-radioactive rapid culture systems, such as the mycobacterial growth indicator tube (MGIT), may prove to be more appropriate in the future for cost-effective TB diagnosis in developing countries.

\section{Acknowledgements}

MMV and WR acknowledge the Lady Tata Memorial Trust, Mumbai for providing the research fellowship. The authors acknowledge C. Basavaraju for technical assistance.

\section{References}

1. Chandramuki A. Tuberculous meningitis and its diagnosis by bacteriological, immunological and molecular methods. Neurosci Today 1998;1:38-41

2. Chandramuki A, Lyashchenko K, Kumari HB, Khanna N, Brusasca P, Gourie-Devi M, et al. Detection of antibody to
Mycobacterium tuberculosis protein antigens in the cerebrospinal fluid of patients with tuberculous meningitis. J Infect Dis 2002;186:678-83.

3. Tortoli E, Cichero P, Chirillo MG, Gismondo MR, Bono L, Gesu G, et al. Multicenter comparison of ESP Culture System II with BACTEC 460TB and with Lowenstein-Jensen medium for recovery of mycobacteria from different clinical specimens, including blood. J Clin Microbiol 1998;36:1378-81.

4. Harris G, Rayner A, Blair J, Watt B. Comparison of three isolation systems for the culture of mycobacteria from respiratory and non-respiratory samples. J Clin Pathol 2000;53:615-8.

5. Roggenkamp A, Hornef MW, Masch A, Aigner B, Autenrieth IB, Heesemann J. Comparison of MB/BacT and BACTEC 460 TB systems for recovery of mycobacteria in a routine diagnostic laboratory. J Clin Microbiol 1999;37:3711-2.

6. Venkataraman P, Herbert D, Paramasivan CN. Evaluation of the BACTEC radiometric method in the early diagnosis of tuberculosis. Indian J Med Res 1998;108:120-7.

7. Chiang TM. Comparison of BACTEC radiometric system and Lowenstein-Jensen medium for detection of Mycobacterium tuberculosis and the susceptibility of isolates to anti-tuberculosis drugs. Zhonghua Yi Xue Za Zhi (Taipei) 1991;48:201-9.

8. Middlebrook G, Reggiardio Z, Tigertt WD. Automatable radiometric detection of growth of Mycobacterium tuberculosis in selective media. Am Rev Respir Dis 1977;115:1067-9.

9. Piersimoni C, Scarparo C, Callegaro A, Tosi CP, Nista D, Bornigiga $\mathrm{S}$, et al. Comparison of $\mathrm{MB} / \mathrm{Bact}$ alert 3D system with radiometric BACTEC system and Lowenstein-Jensen medium for recovery and identification of mycobacteria from clinical specimens: A multicenter study. J Clin Microbiol 2001;39:651-7.

10. Stager CE, Libonati JP, Siddiqi SH, Davis JR, Hooper NM, Baker JF, et al. Role of solid media when used in Conjunction with the BACTEC System for Mycobacterial Isolation and Identification. J Clin Microbiol 1991;29:154-7.

11. Kirihara JM, Hillier SL, Coyle MB. Improved detection times for Mycobacterium avium complex and Mycobacterium tuberculosis with the BACTEC radiometric system. J Clin Microbiol 1985;22:841-5.

12. Wilson ML, Stone BL, Hildred MV, Reves RR. Comparison of recovery rates for mycobacteria from BACTEC 12B vials, Middlebrook and 7H11- selective 7H11 Biplates and LowensteinJensen Slants in a Public Health Mycobacteriology laboratory. $J$ Clin Microbiol 1995;3:2516-8.

13. Salman H. Siddiqi. BACTEC 460TB system- Product and Procedure Manual. 1996.

14. Laszlo A, Siddiqi SH. Evaluation of a rapid radiometric differentiation test for the Mycobacterium tuberculosis complex by selective inhibition with p-nitro alpha-acetylamino-betahydroxypropiophenone. J Clin Microbiol 1984;19:694-8.

15. Morgan MA, Horstmeier CD, DeYoung DR, Roberts GD. Comparison of a radiometric method (BACTEC) and conventional culture media for recovery of mycobacteria from 
smear-negative specimens. J Clin Microbiol 1983;18:384-8

16. Somoskovi A, Kodmon C, Lantos A, Bártfai Z, Tamási L, Füzy $\mathrm{J}$, et al. Comparison of recoveries of Mycobacterium tuberculosis using the automated BACTEC MGIT 960 System, the BACTEC 460TB System and Lowenstein Jensen Medium. J Clin Microbiol 2000;38:2395-7.

17. Daikos GL, Cleary T, Rodriguez A, Fischl MA. Multidrugresistant tuberculous meningitis in patients with AIDS. Int $J$ Tuberc Lung Dis 2003;7:394-8.

18. Roberts GD, Goodman NL, Heifets L, Larsh HW, Lindner TH,
McClatchy JK, et al. Evaluation of the BACTEC radiometric method for recovery of Mycobacteria and Drug Susceptibility testing of Mycobacterium tuberculosis from Acid-Fast Smearpositive specimens. J Clin Microbiol 1983;18:689-96

19. Hooker JA, Muhindi DW, Amayo EO, Mc'ligeyo SO, Bhatt KM, Odhiambo JA. Diagnostic utility of cerebrospinal fluid studies in patients with clinically suspected tuberculous meningitis. Int $J$ Tuberc Lung Dis 2003;7:787-96.

Source of Support: Nil, Conflict of Interest: None declared. 